\title{
Estimation of Finite Population Mean in Stratified Random Sampling Using Auxiliary Attribute(s) under Non-Response
}

\author{
Aneela Saghir \\ Department of Statistics \\ Quaid-i-Azam University, Islamabad \\ nida_850@yahoo.com \\ Javid Shabbir \\ Department of Statistics \\ Quaid-i-Azam University, Islamabad \\ jsqau@yahoo.com
}

\begin{abstract}
In this paper we propose two combined exponential ratio type estimators for finite population mean utilizing information on the auxiliary attribute(s) under non-response. Expressions for bias and MSE of the proposed estimators are derived up to first order of approximation. An empirical study is carried out to observe the performances of the estimators.
\end{abstract}

Keywords: Combined exponential ratio type estimator, bias, Mean Square Error (MSE), efficiency.

\section{Introduction}

The problem of non-response is very common in surveys and consequently the estimators may produce bias results. Hansen and Hurwitz (1946) considered the problem of estimation of population mean under non-response. They suggested of taking a random sample from non-respondents with some extra sources and efforts. They also suggested an estimator $\bar{y}_{s t}^{*}$ by combining information of respondents at first and at second attempts. It is well recognized that use of the auxiliary information may results in considerable reduction in variance of the estimator. Information on the auxiliary variable and Hansen and Hurwitz (1946) technique is used by several authors to estimate the population mean like Cochran (1977), Rao (1986), Khare and Srivastava (1993, 1997), Okafor and Lee (2000) and Singh and Kumar (2008). There are many situations when the auxiliary information is available in form of the auxiliary attributes (see Shabbir and Gupta, 2010). For example the study variable $(y)$ is the production of wheat crop in a certain region, the auxiliary attribute $\left(\psi_{1}\right)$ is the irrigation status (adequate vs. inadequate) and the auxiliary attribute $\left(\psi_{2}\right)$ is the status of land ownership (rich vs. poor).

Consider a finite population of size $N$ and is divided into $L$ strata such that $\sum_{h=1}^{L} N_{h}=N$, where $N_{h}$ is size of the $h^{\text {th }}$ stratum $(h=1,2, \ldots ., L)$. We select a sample of size $n_{h}$ from each stratum by simple random sample without replacement 
sampling such that $\sum_{h=1}^{L} n_{h}=n$, where $n_{h}$ is the stratum sample size. Let $y_{h i}$ and $\psi_{j h i},(j=1,2),\left(i=1,2, \ldots . N_{h}\right)$ be the values of the study variable $(y)$ and the auxiliary attributes $\left(\psi_{j}\right),(j=1,2)$, respectively in the $h^{\text {th }}$ stratum. It is assumed that $n_{h 1}$ units will respond and $n_{h 2}$ will not such that $n_{h 1}+n_{h 2}=n_{h}$. We select a sub-sample of size $r_{h}=\frac{n_{h 2}}{k_{h}}\left(k_{h} \geq 1\right)$ from $n_{h 2}$ units and it is assumed that all the selected units will respond.

We assume that

$\psi_{j h i}=\left\{\begin{array}{cc}1, & \text { if } i^{\text {th }} \text { unit in the stratum } h \text { possesses the attribute } \psi_{j} \quad(j=1,2) \\ 0, & \text { otherwise. }\end{array}\right.$

Let $P_{j}=\sum_{h=1}^{L} W_{h} P_{j h}$ and $p_{j s t}=\sum_{h=1}^{L} W_{h} p_{j h},(j=1,2)$ be the population and sample proportions of units of the auxiliary attributes respectively where $P_{j h}=\frac{A_{j h}}{N_{h}}$, $p_{j h}=\frac{a_{j h}}{n_{h}}, A_{j h}=\sum_{i=1}^{N_{h}} \psi_{j h i}$ and $a_{j h}=\sum_{i=1}^{n_{h}} \psi_{j h i}$.

Let $S_{y h}^{2}=\frac{\sum_{i=1}^{N_{h}}\left(y_{h i}-\bar{Y}_{h}\right)^{2}}{N_{h}-1}$ and $S_{\psi_{j} h}^{2}=\frac{\sum_{i=1}^{N_{h}}\left(\psi_{j h i}-P_{j h}\right)^{2}}{N_{h}-1}$ be the population variances of the study variable and the auxiliary attributes, respectively in the $h^{\text {th }}$ stratum, where $\bar{Y}_{h}=\sum_{i=1}^{N_{h}} y_{h i} / N_{h}$. Let $S_{y \psi_{j} h}=\frac{\sum_{i=1}^{N_{h}}\left(y_{h i}-\bar{Y}_{h}\right)\left(\psi_{j h i}-P_{j h}\right)}{N_{h}-1}$ and $\rho_{y \psi_{j} h}=\frac{S_{y \psi_{j} h}}{S_{\psi_{j} h} S_{y h}}$ be the population bi-covariance and point bi-serial correlation coefficient between the study variable and the auxiliary attributes respectively in the $h^{\text {th }}$. Also $S_{\psi_{1} \psi_{2} h}=\frac{\sum_{i=1}^{N_{h}}\left(\psi_{1 h i}-P_{1 h}\right)\left(\psi_{2 h i}-P_{2 h}\right)}{N_{h}-1}$ and $\rho_{\psi_{1} \psi_{2} h}=\frac{S_{\psi_{1} \psi_{2} h}}{S_{\psi_{1} h} S_{\psi 2 h}}$ be the population phicovariance and phi-correlation coefficient between the auxiliary attributes, respectively in the $h^{\text {th }}$ stratum.

To estimate $\bar{Y}=\sum_{h=1}^{L} W_{h} \bar{Y}_{h}$, we assume that $P_{j h} \quad(j=1,2)$ are known. 
Now adapting the Hansen-Hurwitz methodology, an unbiased estimator of population mean $\bar{Y}$ is given by $\bar{y}_{s t}^{*}=\sum_{h=1}^{L} W_{h} \bar{y}_{h}^{*}$, where $W_{h}=\frac{N_{h}}{N}$ is the known stratum weight and $\bar{y}_{h}^{*}=\frac{n_{h 1} \bar{y}_{1 h}+n_{h 2} \bar{y}_{2 r h}}{n_{h}}$. Here $\bar{y}_{1 h}$ and $\bar{y}_{2 r h}$ are the sample means of $n_{h 1}$ responding units and $n_{h 2}$ sub-sampled units respectively.

The variance of $\bar{y}_{s t}^{*}$ is given by

$\operatorname{Var}\left(\bar{y}_{s t}^{*}\right)=\sum_{h=1}^{L} W_{h}^{2} \gamma_{h} S_{y h}^{2}+\sum_{h=1}^{L} W_{h}^{2} \frac{\left(k_{h}-1\right)}{n_{h}} W_{h 2} S_{y h 2}^{2}$,

where $W_{h 2}=\frac{N_{h 2}}{N_{h}}$ is the known non-response rate in the $h^{\text {th }}$ stratum and $\gamma_{h}=\left(\frac{1}{n_{h}}-\frac{1}{N_{h}}\right)$.

Now we discuss various estimators available in the literature.

The usual combined ratio estimator when the auxiliary attribute is used, given by $\hat{\bar{Y}}_{R_{1} C}=\bar{y}_{s t}^{*} \frac{P_{1}}{p_{1 s t}}$,

where $P_{1}=\sum_{h=1}^{L} W_{h} P_{1 h}$ is known and $p_{1 s t}=\sum_{h=1}^{L} W_{h} p_{1 h}$.

The bias and MSE of $\hat{\bar{Y}}_{R_{1} C}$, to the first degree of approximation, are given by

$\operatorname{Bias}\left(\hat{\bar{Y}}_{R_{1} C}\right) \cong \frac{1}{P_{1}} \sum_{h=1}^{L} W_{h}^{2} \gamma_{h}\left(R_{1} S_{y \psi_{1} h}^{2}-\rho_{y \psi_{1} h} S_{y h} S_{\psi_{1} h}\right)$,

where $R_{1}=\frac{\bar{Y}}{P_{1}}$ and

$$
\operatorname{MSE}\left(\hat{\bar{Y}}_{R_{1} C}\right) \cong \sum_{h=1}^{L} W_{h}^{2} \gamma_{h}\left(S_{y h}^{2}+R_{1}^{2} S_{\psi_{1} h}^{2}-2 R_{1} \rho_{y \psi_{1} h} S_{y h} S_{\psi_{1} h}\right)+\sum_{h=1}^{L} W_{h}^{2} \frac{\left(k_{h}-1\right)}{n_{h}} W_{h 2} S_{y h 2}^{2} \text {. }
$$

Bahl and Tuteja (1991) exponential ratio type estimator when a single auxiliary attribute is used, given by

$\hat{\bar{Y}}_{B T_{1}(R C)}=\bar{y}_{s t}^{*} \exp \left(\frac{P_{1}-p_{1 s t}}{P_{1}+p_{1 s t}}\right)$.

The bias and MSE of $\hat{\bar{Y}}_{B T_{1}(R C)}$, to the first degree of approximation, are given by

$$
\operatorname{Bias}\left(\hat{\bar{Y}}_{B T_{1}(R C)}\right) \cong \frac{1}{2 P_{1}} \sum_{h=1}^{L} W_{h}^{2} \gamma_{h}\left(\frac{3}{4} R_{1} S_{\psi_{1} h}^{2}-\rho_{y \psi_{1} h} S_{y h} S_{\psi_{1} h}\right)
$$

and

$$
\operatorname{MSE}\left(\hat{\bar{y}}_{B T_{1}(R C)}\right) \cong \sum_{h=1}^{L} W_{h}^{2} \gamma_{h}\left(S_{y h}^{2}+\frac{R_{1}^{2}}{4} S_{\psi_{1} h}^{2}-R_{1} \rho_{y \psi_{1} h} S_{y h} S_{\psi_{1} h}\right)+\sum_{h=1}^{L} W_{h}^{2} \frac{\left(k_{h}-1\right)}{n_{h}} W_{h 2} S_{y h 2}^{2} \text {. }
$$


Now we propose the exponential ratio-type estimator for population mean.

\section{Proposed estimators}

Using Bahl and Tuteja (1991) estimator given in (1.5), can be rewritten as

$\hat{\bar{Y}}_{B T_{1}(R C)}=\bar{y}_{s t}^{*} \exp \left(1-\frac{2 p_{1 s t}}{P_{1}+p_{1 s t}}\right)$.

The above estimator can be generalized by introducing a positive real constant ' $a$ ' (i.e. $a \geq 0$ ) instead of ' 2 ' as

$$
\begin{aligned}
& \hat{\bar{Y}}_{P_{1}(R C)}=\bar{y}_{s t}^{*} \exp \left(1-\frac{a p_{1 s t}}{P_{1}+(a-1) p_{1 s t}}\right)=\bar{y}_{s t}^{*} \exp \left(\frac{P_{1}-p_{1 s t}}{P_{1}+(a-1) p_{1 s t}}\right) \text { or } \\
& \hat{\bar{Y}}_{P_{1}(R C)}=\bar{Y}\left(1+e_{0}\right) \exp \left(\frac{P_{1}-P_{1}\left(1+e_{1}\right)}{P_{1}+(a-1) P_{1}\left(1+e_{1}\right)}\right),
\end{aligned}
$$

where $e_{0}=\frac{\bar{y}_{s t}^{*}-\bar{Y}}{\bar{Y}}$ and $e_{1}=\frac{p_{1 s t}-P_{1}}{P_{1}}$.

To first degree of approximation, we have

$$
\hat{\bar{Y}}_{P_{1}(R c)}-\bar{Y} \cong\left(e_{0}-\frac{e_{1}}{a}-\frac{e_{0} e_{1}}{a}+\frac{2 a-1}{2 a^{2}} e_{1}^{2}\right) \text {. }
$$

Using (2.2), the bias and MSE of $\hat{\bar{Y}}_{P_{1}(R C)}$, to the first degree of approximation, are given by

$$
\operatorname{Bias}\left(\hat{\bar{Y}}_{P_{1}(R C)}\right) \cong \frac{R_{1}}{2 a^{2} P_{1}} \sum_{h=1}^{L} W_{h}^{2} \gamma_{h}\left((2 a-1) S_{\psi_{1} h}^{2}-\frac{2 a}{R_{1}} \rho_{y \psi_{1} h} S_{y h} S_{\psi_{1} h}\right)
$$

and

$$
\operatorname{MSE}\left(\hat{\bar{Y}}_{P_{1}(R C)}\right) \cong \sum_{h=1}^{L} W_{h}^{2} \gamma_{h}\left(S_{y h}^{2}+\frac{R_{1}^{2}}{a^{2}} S_{\psi_{1} h}^{2}-2 \frac{R_{1}}{a} \rho_{y \psi_{1} h} S_{\psi_{1} h} S_{y h}\right)+\sum_{h=1}^{L} W_{h}^{2} \frac{k_{h}-1}{n_{h}} W_{h 2} S_{y h 2}^{2} .
$$

Using (2.4), the optimal value of $a$ is given by

$$
a=\frac{R_{1} \sum_{h=1}^{L} W_{h}^{2} \gamma_{h} S_{\psi_{1} h}^{2}}{\sum_{h=1}^{L} W_{h}^{2} \gamma_{h} S_{\psi_{1} h} S_{y h} \rho_{y \psi_{1} h}}=a_{o p t} \text { (say). }
$$

Substituting the optimum value of $a$ in (2.4), we get

$$
\operatorname{MSE}\left(\hat{\bar{Y}}_{P_{1}(R C)}\right)_{o p t} \cong \sum_{h=1}^{L} W_{h}^{2}\left(\gamma_{h} S_{y h}^{2}\left(1-\rho_{y \psi_{1}(s t)}^{2}\right)+\frac{\left(k_{h}-1\right)}{n_{h}} W_{h 2} S_{y h 2}^{2}\right)
$$

where $\rho_{y \psi_{1}(s t)}=\frac{\sum_{h=1}^{L} W_{h}^{2} \gamma_{h} S_{\psi_{1} h} S_{y h} \rho_{y \psi_{1} h}}{\sqrt{\sum_{h=1}^{L} W_{h}^{2} \gamma_{h} S_{y h}^{2}} \sqrt{\sum_{h=1}^{L} W_{h}^{2} \gamma_{h} S_{\psi_{1} h}^{2}}}$ is the combined correlation coefficient between the study variable $(y)$ and the auxiliary attribute $\left(\psi_{1}\right)$. 
An improved exponential ratio type estimator when using two auxiliary attributes is given by

$$
\hat{\bar{Y}}_{P_{2}(R C)}=\bar{y}_{s t}^{*} \exp \left(\frac{P_{1}-p_{1 s t}}{P_{1}+(a-1) p_{1 s t}}\right) \exp \left(\frac{P_{2}-p_{2 s t}}{P_{2}+(b-1) p_{2 s t}}\right) \text {, }
$$

where $a$ and $b$ are positive real constants (i.e. $a \geq 0, b \geq 0$ ) or

$$
\hat{\bar{Y}}_{P_{2}(R C)}=\bar{Y}\left(1+e_{0}\right) \exp \left(\frac{P_{1}-P_{1}\left(1+e_{1}\right)}{P_{1}+(a-1) P_{1}\left(1+e_{1}\right)}\right) \exp \left(\frac{P_{2}-P_{2}\left(1+e_{2}\right)}{P_{2}+(b-1) P_{2}\left(1+e_{2}\right)}\right) \text {, }
$$

where $e_{2}=\frac{p_{2 s t}-P_{2}}{P_{2}}$.

To first degree of approximation, we have

$$
\hat{\bar{Y}}_{P_{2}(R C)}-\bar{Y} \cong\left(e_{0}-\frac{e_{1}}{a}-\frac{e_{2}}{b}-\frac{e_{0} e_{1}}{a}-\frac{e_{0} e_{2}}{b}+\frac{e_{1} e_{2}}{a b}+\frac{2 a-1}{a^{2}} e_{1}^{2}+\frac{2 b-1}{b^{2}} e_{2}^{2}\right)
$$

Using (2.7), the bias and MSE of $\hat{\bar{Y}}_{P_{2}(R C)}$, are given by

$$
\begin{aligned}
& \operatorname{Bias}\left(\hat{\bar{Y}}_{P_{2}(R C)}\right)=\bar{Y} \sum_{h=1}^{L} W_{h}^{2} \gamma_{h}\left(\frac{2 a-1}{2 a^{2}} \frac{S_{\psi_{1} h}^{2}}{P_{1}^{2}}+\frac{2 b-1}{2 b^{2}} \frac{S_{\psi_{2} h}^{2}}{P_{2}^{2}}-\frac{\rho_{y \psi_{1} h} S_{y h} S_{\psi_{1} h}}{a \bar{Y} P_{1}}-\frac{\rho_{y \psi_{2} h} S_{y h} S_{\psi_{2} h}}{b \bar{Y} P_{2}}\right. \\
& \left.+\frac{\rho_{\psi_{1} \psi_{2} h} S_{\psi_{1} h} S_{\psi_{2} h}}{a b P_{1} P_{2}}\right)
\end{aligned}
$$

and

$$
\begin{aligned}
& \operatorname{MSE}\left(\hat{\bar{Y}}_{P_{2}(R C)}\right) \cong \sum_{h=1}^{L} W_{h}^{2} \gamma_{h}\left(S_{y h}^{2}+\frac{R_{1}^{2}}{a^{2}} S_{\psi_{1} h}^{2}+\frac{R_{2}^{2}}{b^{2}} S_{\psi_{2} h}^{2}-2 \frac{R_{1}}{a} \rho_{y \psi_{1} h} S_{y h} S_{\psi_{1} h}-2 \frac{R_{2}}{b} \rho_{y \psi_{2} h} S_{y h} S_{\psi_{2} h}\right. \\
& \left.+2 \frac{R_{1} R_{2}}{a b} \rho_{\psi_{1} \psi_{2} h} S_{\psi_{1} h} S_{\psi_{2} h}\right)+\sum_{h=1}^{L} W_{h}^{2} \frac{k_{h}-1}{n_{h}} W_{h 2} S_{y h 2}^{2} .
\end{aligned}
$$

Using (2.9), the optimal values of $a$ and $b$ are

$$
a_{o p t}=\frac{R_{1} \sum_{h=1}^{L} W_{h}^{2} \gamma_{h} S_{\psi_{1} h}^{2} \sum_{h=1}^{L} W_{h}^{2} \gamma_{h} S_{\psi_{2} h}^{2}\left(1-\rho_{\psi_{1} \psi_{2}(s t)}^{2}\right)}{\sum_{h=1}^{L} W_{h}^{2} \gamma_{h} S_{\psi_{2} h}^{2} \sum_{h=1}^{L} W_{h}^{2} \gamma_{h} \rho_{y \psi_{1} h} S_{y h} S_{\psi_{1} h}-\sum_{h=1}^{L} W_{h}^{2} \gamma_{h} \rho_{y \psi_{2} h} S_{y h} S_{\psi_{2} h} \sum_{h=1}^{L} W_{h}^{2} \gamma_{h} \rho_{\psi_{1} \psi_{2} h} S_{\psi_{1} h} S_{\psi_{2} h}}
$$

and

$$
b_{o p t}=\frac{R_{2} \sum_{h=1}^{L} W_{h}^{2} \gamma_{h} S_{\psi_{1} h}^{2} \sum_{h=1}^{L} W_{h}^{2} \gamma_{h} S_{\psi_{2} h}^{2}\left(1-\rho_{\psi_{1} \psi_{2}(s t)}^{2}\right)}{\sum_{h=1}^{L} W_{h}^{2} \gamma_{h} S_{\psi_{1} h}^{2} \sum_{h=1}^{L} W_{h}^{2} \gamma_{h} \rho_{y \psi_{2} h} S_{y h} S_{\psi_{2} h}-\sum_{h=1}^{L} W_{h}^{2} \gamma_{h} \rho_{y \psi_{1} h} S_{y h} S_{\psi_{1} h} \sum_{h=1}^{L} W_{h}^{2} \gamma_{h} \rho_{\psi_{1} \psi_{2} h} S_{\psi_{1} h} S_{\psi_{2} h}} .
$$

Substituting the optimum values of $a$ and $b$ in (2.9), we get

$$
\operatorname{MSE}\left(\hat{\bar{Y}}_{P_{2}(R C)}\right)_{o p t} \cong \sum_{h=1}^{L} W_{h}^{2}\left(\gamma_{h} S_{y h}^{2}\left\{1-R_{y \cdot \psi_{1} \psi_{2}(s t)}^{2}\right\}+\frac{k_{h}-1}{n_{h}} W_{h 2} S_{y h 2}^{2}\right) \text {, }
$$


where $R_{y \cdot \psi_{1} \psi_{2}(s t)}^{2}=\frac{\rho_{y \psi_{1}(s t)}^{2}+\rho_{y \psi_{2}(s t)}^{2}-2 \rho_{y \psi_{1}(s t)} \rho_{y \psi_{2}(s t)} \rho_{\psi_{1} \psi_{2}(s t)}}{1-\rho_{\psi_{1} \psi_{2}(s t)}^{2}}$ is the combined multiple correlation coefficient of $y$ on $\psi_{1}$ and $\psi_{2}$.

\section{Comparison of estimators}

Now we compare the proposed estimators with other existing estimators.

(i) By (1.1) and (2.5)

$$
\begin{aligned}
& \operatorname{MSE}\left(\hat{\bar{Y}}_{P_{1}(R C)}\right)_{o p t} \leq \operatorname{Var}\left(\bar{y}_{s t}^{*}\right) \text {, if } \\
& \rho_{y \psi_{1}(s t)}^{2} \sum_{h=1}^{L} W_{h}^{2} \gamma_{h} S_{y h}^{2} \geq 0 .
\end{aligned}
$$

(ii) $\quad$ By (1.4) and (2.5)

$$
\begin{aligned}
& \operatorname{MSE}\left(\hat{\bar{Y}}_{P_{1}(R C)}\right)_{o p t} \leq \operatorname{MSE}\left(\hat{\bar{Y}}_{R_{1} C}\right), \text { if } \\
& \left\{\sum_{h=1}^{L} W_{h}^{2} \gamma_{h}\left(R_{1} S_{\psi_{1} h}-\rho_{y \psi_{1} h} S_{y h}\right)\right\}^{2} \geq 0 .
\end{aligned}
$$

(iii) By (1.7) and (2.5)

$$
\begin{aligned}
& \operatorname{MSE}\left(\hat{\bar{Y}}_{P_{1}(R C)}\right)_{o p t} \leq \operatorname{MSE}\left(\hat{\bar{Y}}_{B T_{1}(R C)}\right), \text { if } \\
& \left\{\sum_{h=1}^{L} W_{h}^{2} \gamma_{h}\left(\frac{R_{1}}{2} S_{\psi_{1} h}-\rho_{y \psi_{1} h} S_{y h}\right)\right\}^{2} \geq 0 .
\end{aligned}
$$

(iv) By (1.1) and (2.10)

$$
\begin{aligned}
& \operatorname{MSE}\left(\hat{\bar{Y}}_{P_{2}(R C)}\right)_{o p t} \leq \operatorname{Var}\left(\bar{y}_{s t}^{*}\right), \text { if } \\
& R_{y \cdot \psi_{1} \psi_{2}(s t)}^{2} \sum_{h=1}^{L} W_{h}^{2} \gamma_{h} S_{y h}^{2} \geq 0 .
\end{aligned}
$$

(v) By (1.4) and (2.10)

$$
\begin{aligned}
& \operatorname{MSE}\left(\hat{\bar{Y}}_{P_{2}(R C)}\right)_{o p t} \leq \operatorname{MSE}\left(\hat{\bar{Y}}_{R_{1} C}\right) \text {, if } \\
& \frac{\left(\sum_{h=1}^{L} W_{h}^{2} \gamma_{h}\left\{R_{1}^{2} S_{\psi_{1} h}^{2}-\rho_{y \psi_{h} h} S_{y h} S_{\psi_{1} h}\right\}\right)^{2}}{\sum_{h=1}^{L} W_{h}^{2} \gamma_{h} S_{\psi_{1} h}^{2}}+\frac{\sum_{h=1}^{L} W_{h}^{2} \gamma_{h} S_{y h}^{2}\left(\rho_{y \psi_{1}(s t)} \rho_{\psi_{1} \psi_{2}(s t)}-\rho_{y \psi_{2}(s t)}\right)^{2}}{1-\rho_{\psi_{1} \psi_{2}(s t)}^{2}} \geq 0 .
\end{aligned}
$$

(vi) By (1.7) and (2.10)

$$
\begin{aligned}
& \operatorname{MSE}\left(\hat{\bar{Y}}_{P_{2}(R C)}\right)_{o p t} \leq \operatorname{MSE}\left(\hat{\bar{Y}}_{B T_{1} C}\right) \text {, if } \\
& \frac{\left(\sum_{h=1}^{L} W_{h}^{2} \gamma_{h}\left\{\frac{R_{1}^{2} S_{\psi_{1} h}^{2}}{2}-\rho_{y \psi_{1} h} S_{y h} S_{\psi_{1} h}\right\}\right)^{2}}{\sum_{h=1}^{L} W_{h}^{2} \gamma_{h} S_{\psi_{1} h}^{2}}+\frac{\sum_{h=1}^{L} W_{h}^{2} \gamma_{h} S_{y h}^{2}\left(\rho_{y \psi_{1}(s t)} \rho_{\psi_{1} \psi_{2}(s t)}-\rho_{y \psi_{2}(s t)}\right)^{2}}{1-\rho_{\psi_{1} \psi_{2}(s t)}^{2}} \geq 0 .
\end{aligned}
$$


(vii) By (2.5) and (2.10)

$$
\begin{aligned}
& \operatorname{MSE}\left(\hat{\bar{Y}}_{P_{2}(R C)}\right)_{o p t} \leq \operatorname{MSE}\left(\hat{\bar{Y}}_{P_{1}(R C)}\right)_{o p t} \text {, if } \\
& \frac{\sum_{h=1}^{L} W_{h}^{2} \gamma_{h} S_{y h}^{2}\left(\rho_{y \psi_{1}(s t)} \rho_{\psi_{1} \psi_{2}(s t)}-\rho_{y \psi_{2}(s t)}\right)^{2}}{1-\rho_{\psi_{1} \psi_{2}(s t)}^{2}} \geq 0 .
\end{aligned}
$$

The above conditions in (3.1)-(3.7) are obviously true.

\section{Numerical Analysis}

Source: [Murthy (1967)]

We randomly select a sample of size $n_{h}$ from each stratum by using the Neyman allocation and consider the first $10 \%, 20 \%$ and $30 \%$ values in each stratum as non-response for $W_{h 2}=0.1, W_{h 2}=0.2$ and $W_{h 3}=0.3$ respectively.

The population consists of village wise complete enumeration and data are obtained in 1951 and 1961 censuses for a Tehsil. The area of village is used to stratify the population into three strata.

Let $y$ be the cultivated area in the village in hectares in 1951 and $\psi_{j h i} \quad(j=1,2)$ are defined below.

Let $\psi_{11 i}=\left\{\begin{array}{lc}1, & \text { if } i^{t h} \text { village in the Stratum } 1 \text { has area greater than } 550 \text { hectares } \\ 0, & \text { otherwise. }\end{array}\right.$

$$
\psi_{12 i}=\left\{\begin{array}{cc}
1, & \text { if } i^{t h} \text { village in the Stratum } 2 \text { has area greater than } 1300 \text { hectares } \\
0, & \text { otherwise. }
\end{array}\right.
$$

and $\psi_{13 i}=\left\{\begin{array}{lc}1, & \text { if } i^{\text {th }} \text { village in the Stratum } 3 \text { has area greater than } 2500 \text { hectares } \\ 0, & \text { otherwise. }\end{array}\right.$

Let

$\psi_{21 i}=\left\{\begin{array}{lc}1, & \text { if } i^{t h} \text { village in the Stratum } 1 \text { has number of cultivators greator then } 450 \\ 0, & \text { otherwise }\end{array}\right.$

$\psi_{22 i}=\left\{\begin{array}{lc}1, & \text { if } i^{t h} \text { village in the Stratum } 2 \text { has number of cultivators greator then } 700 \\ 0, & \text { otherwise. }\end{array}\right.$

and

$\psi_{23 i}=\left\{\begin{array}{lc}1, & \text { if } i^{\text {th }} \text { village in the Stratum } 3 \text { has number of cultivators greator then } 1500 \\ 0, & \text { otherwise. }\end{array}\right.$ 
Data are presented in Table 4.1 and results are given in Table 4.2.

Table 4.1: Data Statistics

\begin{tabular}{|c|c|c|c|c|}
\hline \multicolumn{2}{|c|}{ Stratum No. } & 1 & 2 & 3 \\
\hline \multicolumn{2}{|l|}{$N_{h}$} & 43 & 45 & 40 \\
\hline \multicolumn{2}{|l|}{$n_{h}$} & 10 & 12 & 18 \\
\hline \multicolumn{2}{|l|}{$\bar{Y}_{h}$} & 397.1425 & 760.1795 & 1234.6180 \\
\hline \multicolumn{2}{|l|}{$P_{1 h}$} & 0.5814 & 0.4444 & 0.4000 \\
\hline \multicolumn{2}{|l|}{$P_{2 h}$} & 0.39535 & 0.5333 & 0.4000 \\
\hline \multicolumn{2}{|l|}{$S_{y h}^{2}$} & 39975.0569 & 61455.990 & 172425.900 \\
\hline \multicolumn{2}{|l|}{$S_{\psi_{1} h}^{2}$} & 0.2492 & 0.2525 & 0.2462 \\
\hline \multicolumn{2}{|l|}{$S_{\psi_{2} h}^{2}$} & 0.2448 & 0.2545 & 0.2462 \\
\hline \multicolumn{2}{|c|}{$\rho_{y \psi_{1} h}$} & 0.6922 & 0.3750 & 0.5057 \\
\hline \multicolumn{2}{|c|}{$\rho_{y \psi_{2} h}$} & 0.3956 & 0.2847 & 0.3261 \\
\hline \multicolumn{2}{|c|}{$\rho_{\psi_{1} \psi_{2} h}$} & 0.2040 & 0.3884 & 0.5833 \\
\hline \multirow{3}{*}{$S_{y h 2}^{2}$} & For $W_{h 2}=0.1$ & 16871.6298 & 5534.3610 & 174694.200 \\
\hline & For $W_{h 2}=0.2$ & 10951.1712 & 43003.2000 & 123887.767 \\
\hline & For $W_{h 2}=0.3$ & 15878.3587 & 49346.8000 & 152450.527 \\
\hline
\end{tabular}

We compute the percent relative efficiency (PRE) of different estimators for different values of $W_{h 2}$ and $k_{h}$ and use the following expression

$\operatorname{PRE}=\frac{\operatorname{Var}\left(\bar{y}_{s t}^{*}\right)}{\operatorname{MSE}\left(\hat{\bar{Y}}_{i}\right)} \times 100$, where $i=P_{1}(R C), B T_{1}(R C), R_{1} C, P_{2}(R C)$ 
Table 4.2: Percentage relative efficiency of different estimators with respect to $\bar{y}_{s t}^{*}$

\begin{tabular}{|c|c|c|c|c|c|c|c|c|}
\hline \multirow[t]{2}{*}{$W_{h 2}$} & \multirow[t]{2}{*}{$k_{h}$} & \multicolumn{2}{|c|}{$\operatorname{PRE}\left(\hat{\bar{Y}}_{P_{1}(R C)}, \bar{y}_{s t}^{*}\right)$} & \multicolumn{2}{|c|}{$\operatorname{PRE}\left(\hat{\bar{Y}}_{B T_{1}(R C)}, \bar{y}_{s t}^{*}\right)$} & \multicolumn{2}{|c|}{$\operatorname{PRE}\left(\hat{\bar{Y}}_{R_{1} C}, \bar{y}_{s t}^{*}\right)$} & \multirow{2}{*}{$\begin{array}{c}\operatorname{PRE}\left(\hat{\bar{Y}}_{P_{2}(R C)}, \bar{y}_{s t}^{*}\right) \\
\text { Using first } \\
\text { and second } \\
\text { auxiliary } \\
\text { attributes }\end{array}$} \\
\hline & & $\begin{array}{c}\text { Using } \\
\text { first } \\
\text { auxiliary } \\
\text { attribute }\end{array}$ & $\begin{array}{c}\text { Using } \\
\text { second } \\
\text { auxiliary } \\
\text { attribute }\end{array}$ & $\begin{array}{c}\text { Using } \\
\text { first } \\
\text { auxiliary } \\
\text { attribute }\end{array}$ & $\begin{array}{c}\text { Using } \\
\text { second } \\
\text { auxiliary } \\
\text { attribute }\end{array}$ & $\begin{array}{c}\text { Using } \\
\text { first } \\
\text { auxiliary } \\
\text { attribute }\end{array}$ & $\begin{array}{c}\text { Using } \\
\text { second } \\
\text { auxiliary } \\
\text { attribute }\end{array}$ & \\
\hline \multirow[t]{4}{*}{0.1} & 2 & 129.83 & 110.12 & 54.93 & 38.38 & 13.95 & 10.68 & 133.78 \\
\hline & 2.5 & 128.31 & 109.68 & 55.93 & 39.35 & 14.45 & 11.07 & 132.01 \\
\hline & 3 & 126.94 & 109.27 & 56.89 & 40.28 & 14.94 & 11.46 & 130.42 \\
\hline & 3.5 & 125.69 & 108.90 & 57.80 & 41.19 & 15.42 & 11.84 & 128.97 \\
\hline \multirow[t]{4}{*}{0.2} & 2 & 126.75 & 109.22 & 57.02 & 40.41 & 15.01 & 11.51 & 130.20 \\
\hline & 2.5 & 124.32 & 108.49 & 58.87 & 42.25 & 15.10 & 12.31 & 127.39 \\
\hline & 3 & 122.30 & 107.87 & 60.57 & 43.98 & 16.97 & 13.09 & 125.06 \\
\hline & 3.5 & 120.58 & 107.33 & 62.13 & 45.61 & 17.92 & 13.86 & 123.09 \\
\hline \multirow[t]{4}{*}{0.3} & 2 & 122.93 & 108.33 & 60.02 & 43.64 & 16.65 & 12.87 & 125.98 \\
\hline & 2.5 & 119.82 & 107.31 & 62.86 & 46.62 & 18.39 & 14.28 & 122.38 \\
\hline & 3 & 117.45 & 106.52 & 65.33 & 49.30 & 20.05 & 15.64 & 119.65 \\
\hline & 3.5 & 115.59 & 105.88 & 67.49 & 51.72 & 21.65 & 16.96 & 117.52 \\
\hline
\end{tabular}

From Table 4.2, we observed that the proposed estimators are more efficient as compared to the usual estimator $\bar{y}_{s t}^{*}$ and the other considered estimators. The efficiency of the estimators decreases with increases level of non-response in each stratum and $k_{h}$. As correlation between the study variable and the auxiliary attribute(s) increases in each stratum, the efficiency also increases.

\section{Conclusion}

In this paper, we have proposed two combined exponential ratio type estimators when using information on the auxiliary attribute(s) under non-response. Expressions for bias and MSE of the proposed estimators are derived up to first degree of approximation. The proposed estimators are compared with usual 
mean estimator and other considered estimators. A numerical study is carried out to support the theoretical results for different values of non-responses. In Table 4.2, the proposed estimators perform better then the usual sample mean estimator and other considered estimators. The efficiency of the estimators decreases with increase rate of non-response $W_{h 2}$ and $k_{h}$.

\section{Acknowledgements}

Authors are thankful to the learned referees for their valuable suggestions regarding improvement of the research paper.

\section{References}

1. Bahl, S. and Tuteja, R.K. (1991). Ratio and product type exponential estimator. Information and Optimization Sciences.12, 159-163.

2. Cochran, W.G. (1977). Sampling Techniques, $3^{\text {rd }}$ edn. New York: John Wiley and Sons.

3. Hansen, M.H. and Hurwitz, W.N. (1946). The problem of non-response in sample surveys. Journal of the American Statistical Association. 41, 517529.

4. Khare, B.B. and Srivastava, S. (1993). Estimation of population mean using auxiliary character in presence of non-response. National Academy Science Letters India .16, 111-114.

5. Khare, B.B. and Srivastava, S. (1997). Transformed ratio and product type estimators for the population mean in the presence of non-response. Communication in Statistics-Theory and Methods. 26, 1779-1791.

6. Murthy, M. N. (1967): Sampling Theory and Methods. Statistical Publishing Society, India.

7. Okafor, F.C. and Lee, H. (2000). Double sampling for ratio and regression estimation with sub-sampling the non-respondents. Survey Methodology. 26, 183-188.

8. Rao, P.S.R.S. (1986). Ratio estimation with sub-sampling the nonrespondents. Survey Methodology. 12(2), 217-230.

9. Shabbir, J., Gupta, S. (2010). Estimation of the finite population mean in two phase sampling when auxiliary variables are attributes. Hacettepe Journal of Mathematics and Statistics. 39 (1), 121-129.

10. Singh, H.P. and Kumar, S. (2008). A regression approach to the estimation of the finite population mean in presence of non-response. Australian and New Zealand Journal of Statistics. 50, 395-408. 